Article

\title{
Electrochemical and Spectroelectrochemical Properties of a New Donor-Acceptor Polymer Containing 3,4-Dialkoxythiophene and 2,1,3-Benzothiadiazole Units
}

\author{
Erika Herrera Calderon, Milind Dangate, Norberto Manfredi, Alessandro Abbotto *, \\ Matteo M. Salamone, Riccardo Ruffo * and Claudio M. Mari
}

Milano-Bicocca Solar Energy Research Center-MIB-Solar, Department of Materials Science, University of Milano Bicocca, Via Cozzi 53, Milano I-20125, Italy;

E-Mails: erika.herrera.calderon@gmail.com (E.H.C.); mili_ncl@yahoo.com (M.D.); norberto.manfredi@mater.unimib.it (N.M.); matteo.salamone@mater.unimib.it (M.M.S.); claudiomaria.mari@unimib.it (C.M.M.)

* Authors to whom correspondence should be addressed;

E-Mails: alessandro.abbotto@unimib.it (A.A.); riccardo.ruffo@unimib.it (R.R.);

Tel.: +39-02-6448-5153 (R.R.); Fax: +39-02-6448-5400 (R.R. and A.A.).

Received: 30 May 2013; in revised form: 23 July 2013 / Accepted: 5 August 2013 /

Published: 12 August 2013

\begin{abstract}
A new heteroarylene-vinylene donor-acceptor low bandgap polymer, the poly(DEHT-V-BTD), containing vinylene-spaced efficient donor (dialkoxythiophene) and acceptor (benzothiadiazole) moieties, is presented. Electropolymerization has been carried out by several electrochemical techniques and the results are compared. In particular, the pulsed potentiostatic method was able to provide layers with sufficient amounts of material. Cyclic voltammetries showed reversible behavior towards both $\mathrm{p}$ - and $\mathrm{n}$-doping. The HOMO, LUMO, and bandgap energies were estimated to be $-5.3,-3.6$ and $1.8 \mathrm{eV}$, respectively. In situ UV-Vis measurements have established that the presence of the vinylene group stabilizes the formation of polaronic charge carriers even at high doping levels.
\end{abstract}

Keywords: donor-acceptor-donor monomer; conducting polymer; electropolymerization; electrochemistry; 3,4-bis(2-ethylhexyloxy)thiophene; 2,1,3-benzothiadiazole 


\section{Introduction}

Low bandgap polymers are promising materials for their possible use in a wide range of applications, in particular as donor p-conducting layers in bulk-heterojunction (BHJ) solar cells, wherein they are commonly used in combination with fullerene derivatives such as $[6,6]$-phenyl- $\mathrm{C}_{61}$-butyric acid methyl ester (PCBM) as acceptor n-conducting materials [1]. In such application, the polymer energy levels need to be finely tuned in order to have efficient BHJ properties. In particular, optimized design rules dictate that: (1) the bandgap of the polymer should be equal or lower than $1.8 \mathrm{eV}$ in order to efficiently absorb the solar radiation up to the low energy portion of the spectrum; (2) the energy of the Lowest Unoccupied Molecular Orbital (LUMO) of the polymer should be higher (with a minimal offset of $0.3 \mathrm{eV}$ ) than that of PCBM in order to ensure efficient charge separation; (3) the Highest Occupied Molecular Orbital (HOMO) of the polymer should be as low as possible in order to have a high open circuit voltage, being this proportion to the difference between the energy of the LUMO PCBM and HOMO polymer. In order to reach an optimal trade-off between these requirements, recent design rules suggest that optimal donor polymers should have bandgap energies in the range of 1.3-1.9 eV [1]. One of the most promising approaches to induce these properties is a molecular design of monomeric precursors with donor-acceptor constituting units [2]. The resulting polymer will thus consist of alternating $\pi$-electronrich donor (D) and $\pi$-electronpoor acceptor (A) moieties along the backbone [3-6]. A large variety of D groups have been so far proposed. Amongst these, 3,4-ethylendioxythiophene (EDOT) has been often used due to its outstanding chemical stability and good transport properties [7].

In spite of the large variety of D-A polymers, relatively few examples are known for vinylene-linked (V) conjugated polymers, where the D and A fragments are separated by an ethenylic spacer. Indeed, superior photovoltaic performances in terms of both conductivity and control of the energy levels can be enabled by inserting vinylene spacers into the structure between the D and A groups [8]. We have recently designed and investigated new polymers starting from D-A-D monomers, wherein the D and A units were separated by a vinylene group $[9,10]$. In particular, we have used pyrrole [9] and EDOT [10] as donor heteroaromatic fragments and pyridine as the acceptor units. In these works we have shown how it is possible to reach optimal design requirements for BHJ devices starting from very simple and commonly used heteroaromatic units, with a fine tuning arising from the proper substitution pattern around the central pyridine ring. However, the electrochemical properties of our previous pyridine-based polymers did not show evidence of a full reversible electrochemical reduction process (n-doping). In order to increase the electron-withdrawing character of the central acceptor group, we have decided to design a new vinylene-spaced D-A-D monomer where the pyridine ring has been replaced by the 2,1,3-benzothiadiazole (BTD) ring, which has triggered a considerable interest in recent years as an acceptor unit due to its strong electron-withdrawing capacity and ready availability of commercial precursors [11-13]. As a donor unit we first turned our attention on the EDOT ring. Unfortunately, the corresponding (EDOT-V-BTD) monomer showed poor solubility in most solvents. For this reason, we replaced EDOT with a 3,4-bis(2-ethylhexyloxy)thiophene (DEHT) ring, which has similar electronic properties but a larger solubility in organic solvents and improved filming properties thanks to the presence of two branched side alkyl chains [13]. We have thus designed and prepared the (DEHT-V-BTD) monomer (Figure 1). In the present contribution we 
report the preparation and characterization of the new monomer, its electrochemical polymerization, and the electrochemical and spectroelectrochemical properties of the resulting polymeric layers. In particular, the poly(DEHT-V-BTD) energy levels and the nature of the charge carries formed during the oxidation and the reduction of the layers are discussed in comparison with the poly(2,5-pyridine-Vpyrrole) [9] and poly(2,5-pyridine-V-EDOT) [10].

Figure 1. The monomer (DEHT-V-BTD).

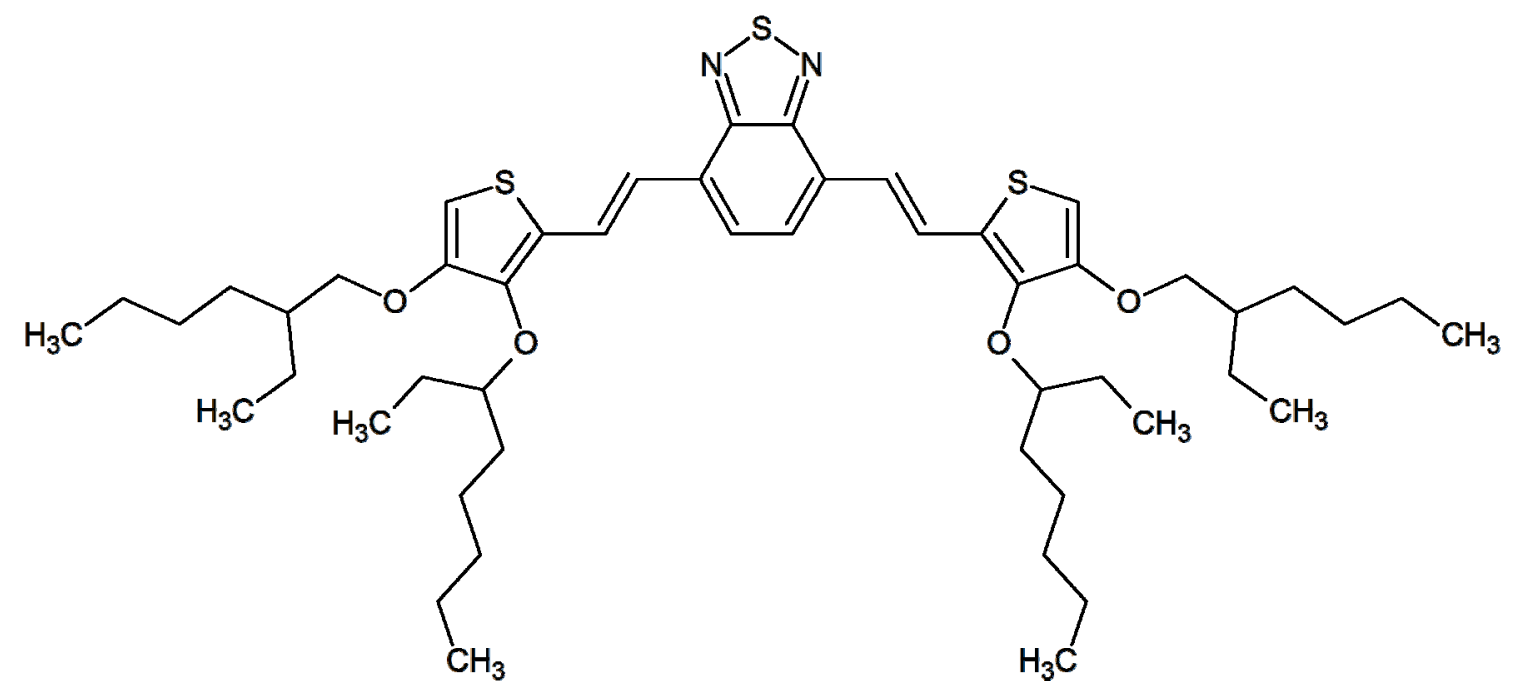

\section{Experimental Section}

\subsection{Monomer Preparation}

All reagents were obtained from commercial suppliers at the highest purity grade and used without further purification, except for $\mathrm{POCl}_{3}$ that was distilled prior to use. Anhydrous solvents were purchased from Sigma Aldrich and used without further purification. Extracts were dried over $\mathrm{Na}_{2} \mathrm{SO}_{4}$ and filtered before the removal of the solvent by evaporation. Flash chromatography was performed with Merck grade 9385 silica gel 230-400 mesh (60 ̊).

3,4-Bis(2-ethylhexyloxy)thiophene-2-carbaldehyde (2). $\mathrm{POCl}_{3}(2.7 \mathrm{~mL}, 4.4 \mathrm{~g}, 29 \mathrm{mmol}$ ) was added dropwise to ice-cold DMF $(1.2 \mathrm{~mL}, 1.1 \mathrm{~g}, 15 \mathrm{mmol})$. The solution was stirred for $20 \mathrm{~min}$ and the obtained white solid was dissolved in dry 1,2-dichloroethane $(60 \mathrm{~mL})$. A solution of 3,4-bis(2-ethylhexyloxy)thiophene (1) [13] (5.00 g, $14.7 \mathrm{mmol})$ in 1,2-dichloroethane (10 mL) was then added dropwise and the mixture refluxed for $1 \mathrm{~h}$. The reaction mixture was allowed to cool to room temperature and then poured onto a saturated ice-cold solution of $\mathrm{AcONa}(50 \mathrm{~mL})$. The resulting mixture was stirred at reflux for $1 \mathrm{~h}$ and then extracted with $\mathrm{CH}_{2} \mathrm{Cl}_{2}(100 \mathrm{~mL})$. The organic layer were thoroughly washed with water, dried, and the solvent was evaporated under vacuum to afford the product as a yellow-brown oil (5.20 g, $14.1 \mathrm{mmol}, 96 \%)$, which was used for the next step without further purification; ${ }^{1} \mathrm{H}-\mathrm{NMR}\left(\mathrm{CDCl}_{3}\right) \delta 9.99(\mathrm{~s}, 1 \mathrm{H}, \mathrm{CHO}), 6.61(\mathrm{~s}, 1 \mathrm{H}$, thiophene), 4.28-4.18 (m, $2 \mathrm{H}$, diastereotopic $\mathrm{OCH}_{2}$ protons), $3.86\left(\mathrm{~d}, \mathrm{~J}=5.9 \mathrm{~Hz}, 2 \mathrm{H}\right.$, diastereotopic $\mathrm{OCH}_{2}$ protons), 1.77-1.63 (m, $2 \mathrm{H}, \mathrm{CH}$ of alkyl chains), 1.56-1.37 (m, 16H, remaining $\mathrm{CH}_{2}$ of alkyl chains), $0.96-0.84\left(\mathrm{~m}, 12 \mathrm{H}, \mathrm{CH}_{3}\right.$ of alkyl chains). 
3,4-Bis(2-ethylhexyloxy)-2-vinylthiophene (3). n-Butyllithium (2.5 M in hexane, 5.6 mL, $14.0 \mathrm{mmol}$ ) was added via syringe to a solution of methyltriphenylphosphonium bromide $(5.00 \mathrm{~g}, 14.0 \mathrm{mmol})$ in THF $(100 \mathrm{~mL})$ at $0{ }^{\circ} \mathrm{C}$. A solution of aldehyde $(2)(5.20 \mathrm{~g}, 14.0 \mathrm{mmol})$ in THF $(20 \mathrm{~mL})$ was added dropwise at $-78^{\circ} \mathrm{C}$ and the resulting mixture was allowed to warm up to room temperature and stirred for $12 \mathrm{~h}$. The mixture was then poured into water $(150 \mathrm{~mL})$ and extracted with $\mathrm{CH}_{2} \mathrm{Cl}_{2}(2 \times 50 \mathrm{~mL})$. The organic layer was washed with brine, dried, and evaporated to dryness to leave a residue which was purified by column chromatography (cyclohexane:dichloromethane $=7: 3$ ) affording the practically pure product as a yellow oil $(4.55 \mathrm{~g}, 12.4 \mathrm{mmol}, 89 \%) ;{ }^{1} \mathrm{H}-\mathrm{NMR}\left(\mathrm{CDCl}_{3}\right) \delta 6.84\left(\mathrm{dd}, \mathrm{J}_{\text {trans }}=17.6 \mathrm{~Hz}\right.$, $\left.\mathrm{J}_{\mathrm{cis}}=11.0 \mathrm{~Hz}, 1 \mathrm{H},\right), 5.99(\mathrm{~s}, 1 \mathrm{H}$, thiophene $), 5.45\left(\mathrm{dd}, \mathrm{J}_{\text {trans }}=17.6 \mathrm{~Hz}, \mathrm{~J}_{\mathrm{gem}}=0.8 \mathrm{~Hz}, 1 \mathrm{H}\right.$, $\beta$-Z-vinyl proton to thiophene), 5.05 (dd, $\mathrm{J}_{\text {cis }}=11.0 \mathrm{~Hz}, \mathrm{~J}_{\mathrm{gem}}=0.8 \mathrm{~Hz}, 1 \mathrm{H}, \beta$-E-vinyl proton to thiophene), 3.99-3.92 (m, 2H, diastereotopic $\mathrm{OCH}_{2}$ protons), 3.87-3.81 (m, $2 \mathrm{H}$, diastereotopic $\mathrm{OCH}_{2}$ protons), 1.77-1.69 (m, 1H, $\mathrm{CH}$ of alkyl chain), 1.69-1.61 (m, 1H, $\mathrm{CH}$ of alkyl chain), 1.61-1.25 (m, $16 \mathrm{H}$, remaining $\mathrm{CH}_{2}$ of alkyl chains), $0.98-0.88$ (m, $12 \mathrm{H}, \mathrm{CH}_{3}$ of alkyl chains).

DEHT-V-BTD. A solution of tris(o-tolyl)phosphine $(7 \mathrm{mg}, 0.024 \mathrm{mmol})$ and $\mathrm{Pd}(\mathrm{OAc})_{2}(1 \mathrm{mg}$, $0.006 \mathrm{mmol})$ in dry DMF $(5 \mathrm{~mL})$ was added dropwise to a solution of 4,7-dibromo-2,1,3benzothiadiazole $(90 \mathrm{mg}, 0.307 \mathrm{mmol})$, derivate $(3)(250 \mathrm{mg}, 0.683 \mathrm{mmol})$, and triethylamine $(0.7 \mathrm{~mL})$ in dry DMF $(20 \mathrm{~mL})$ at $80{ }^{\circ} \mathrm{C}$ under an argon atmosphere. After stirring for $12 \mathrm{~h}$ at $100{ }^{\circ} \mathrm{C}$ the reaction mixture was poured into water $(25 \mathrm{~mL})$ and extracted with $\mathrm{CH}_{2} \mathrm{Cl}_{2}(3 \times 50 \mathrm{~mL})$. The combined organic layers were treated with aqueous $1.2 \mathrm{~N} \mathrm{HCl}(10 \mathrm{~mL})$, washed with brine $(3 \times 10 \mathrm{~mL})$, dried, and evaporated to dryness in vacuum to leave a residue which was purified by column chromatography (cyclohexane:dichloromethane $=7: 3$ ) to give the pure product $(72 \mathrm{mg}, 0.083 \mathrm{mmol}, 27 \%$ ) as a red jelly; ${ }^{1} \mathrm{H}-\mathrm{NMR}\left(\mathrm{CDCl}_{3}\right) \delta 8.28(\mathrm{~d}, \mathrm{~J}=16.2 \mathrm{~Hz}, 2 \mathrm{H}, \beta$-vinyl proton to benzothiadiazole), $7.55(\mathrm{~s}, 2 \mathrm{H}$, benzothiadiazole), $7.28(\mathrm{~d}, \mathrm{~J}=16.2 \mathrm{~Hz}, 2 \mathrm{H}, \alpha$-vinyl proton to benzothiadiazole), $6.12(\mathrm{~s}, 2 \mathrm{H}$, thiophene), $4.08\left(\mathrm{~d}, \mathrm{~J}=9.9 \mathrm{~Hz}, 4 \mathrm{H}\right.$, diastereotopic $\mathrm{OCH}_{2}$ protons $), 3.87(\mathrm{~d}, \mathrm{~J}=5.4 \mathrm{~Hz}, 4 \mathrm{H}$, diastereotopic $\mathrm{OCH}_{2}$ protons), $1.85-1.20$ (m, 36H, $\mathrm{CH}$ and remaining $\mathrm{CH}_{2}$ of alkyl chains), 1.08-0.80 (m, $24 \mathrm{H}, \mathrm{CH}_{3}$ of alkyl chains); HRMS-ESI: $m / z$ calculated for $\mathrm{C}_{50} \mathrm{H}_{77} \mathrm{~N}_{2} \mathrm{O}_{4} \mathrm{~S}_{3}(\mathrm{M}+\mathrm{H})+865.50455$, found 865.50515; calculated for $\mathrm{C}_{50} \mathrm{H}_{76} \mathrm{~N}_{2} \mathrm{NaO}_{4} \mathrm{~S}_{3}(\mathrm{M}+\mathrm{Na})+887.48649$, found 887.4868.

\subsection{Optical Characterization}

Absorption and emission spectra were recorded using a V-570 Jasco spectrophotomer (Jasco, Easton, MD, USA) and a Jasco FP 6200 spectrofluorometer (Jasco, Easton, MD, USA), respectively. The spanned wavelength range was from 300 to $900 \mathrm{~nm}$. The optical bandgaps of the monomer and polymer were calculated on the low energetic edge of the corresponding absorption spectra.

\subsection{Electropolymerization and Electrochemical Characterizations}

Thin films of poly(DEHT-V-BTD) were grown on Fluorine Tin Oxide (FTO, $15 \Omega /$ square) by electrochemical polymerization using cyclic voltammetry (CV), potentiostatic (PS), and pulsed-potentiostatic (PPS) techniques starting from saturated monomer solutions (100 mg in $25 \mathrm{~mL}$ ) in the supporting electrolyte $(0.1 \mathrm{M}$ electrochemical grade tetrabutylammonium hexafluorophosphate in anhydrous acetonitrile). Electrodepositions were performed in a three-electrode cell with FTO 
(area about $1 \mathrm{~cm}^{2}$ ), $\mathrm{Ag} / \mathrm{AgCl}$ wire, and Pt flag, as working, pseudoreference, and counter electrode, respectively. A similar cell was used for the electrochemical characterization (CV) of polymers using the deposed layer as working electrode; all the $\mathrm{CVs}$ were performed at $50 \mathrm{mV} \cdot \mathrm{s}^{-1}$. The $\mathrm{Ag} / \mathrm{AgCl}$ pseudo-reference was calibrated before each measurement using ferrocene solution and all the potentials are reported $v s$. the $\mathrm{Fc}^{+} / \mathrm{Fc}$ couple. All the electrochemical measurements were performed in an $\mathrm{Ar}$ filled glove box $\left(\left[\mathrm{O}_{2}\right]<1 \mathrm{ppm}\right)$. The spectroelectrochemical measurements in the near-infrared/Vis range $(1100 \geq \lambda \geq 340 \mathrm{~nm})$ were performed using the same cell arrangement in a sealed optical cuvette. Each spectrum acquisition was performed under bias potential which was applied before the spectrum acquisition for a time long enough to drive the system into a stationary state. The HOMO-LUMO determination was carried out using the current onset of the stable CV traces [14] using a vacuum level of $-4.5 \mathrm{eV}$ for the Standard Hydrogen Electrode (SHE) [15] and a redox potential of $0.69 \mathrm{~V}$ vs. SHE for the $\mathrm{Fc}^{+} / \mathrm{Fc}$ in $\mathrm{ACN}$ [16].

\section{Results and Discussion}

\subsection{Synthesis and Optical Properties of the Monomer}

The monomer DEHT-V-BTD was obtained according to the synthetic route reported in Figure 2. 3,4-Bis(2-ethylhexyloxy)thiophene [13] (1) was converted by Vilsmeier formylation to the corresponding aldehyde (2), which was then condensed with the methyltriphenylphosphonium bromide to give the 2-vinyl derivative (3). The desired monomer was finally obtained by the Heck palladium-catalyzed cross-coupling reaction between (3) and the 4,7-dibromo-2,1,3-benzothiadiazole.

Figure 2. Synthesis of monomer DEHT-V-BTD.

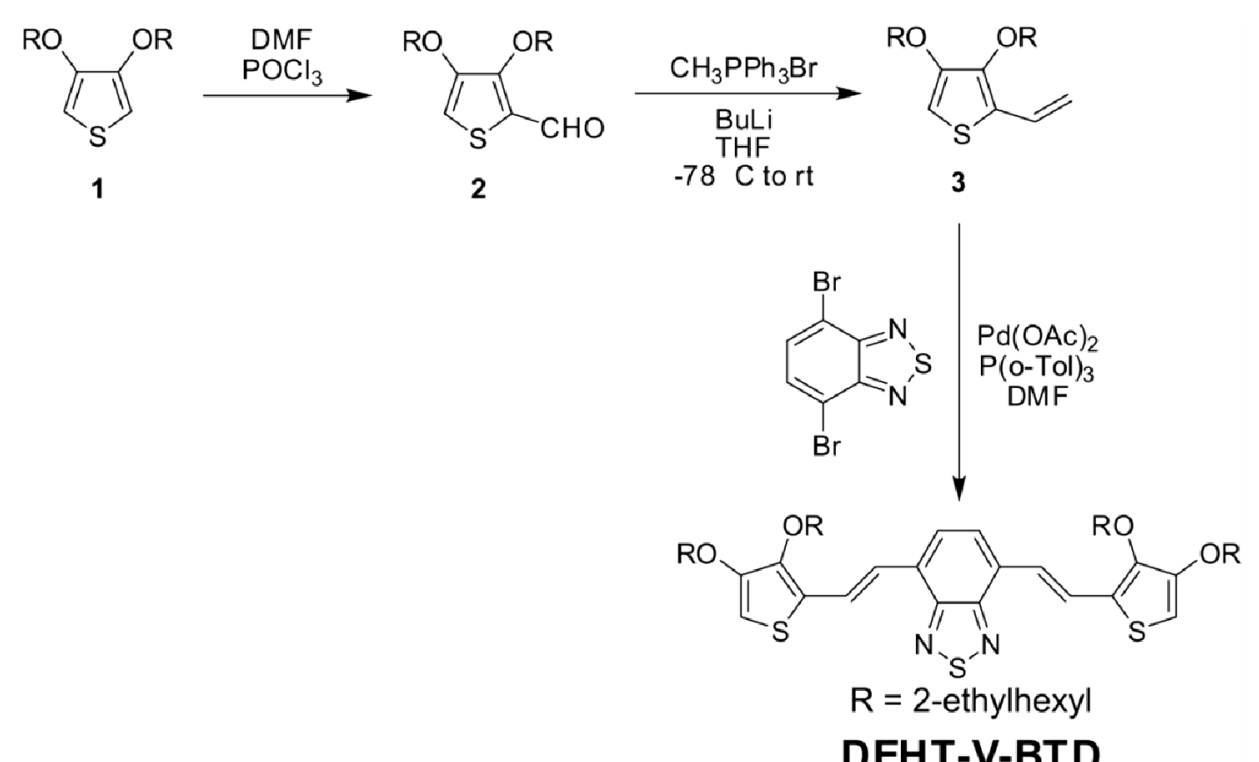

Figure 3 depicts the normalized absorption and emission spectra of the monomer DEHT-V-BTD. The monomer has two absorption bands, one at higher energies (peak at $326 \mathrm{~nm}$ ) related to local transitions of the heteroaromatic units, and one at lower energies (peak at $480 \mathrm{~nm}$ ) associated to the charge transfer (CT) transition from the electron-rich donor thiophene ring to the BTD acceptor. Upon excitation at the latter absorption peak, an emission band was recorded with a maximum at about 
$600 \mathrm{~nm}$. The $0-0$ transition energy $(2.3 \mathrm{eV})$, calculated from the intercept of the normalized absorption and emission spectra, is slightly higher than the corresponding optical band gap (Table 1).

Figure 3. Absorption (continuous line) and emission spectra (dashed line) of monomer DEHT-V-BTD in $\mathrm{CHCl}_{3}$.

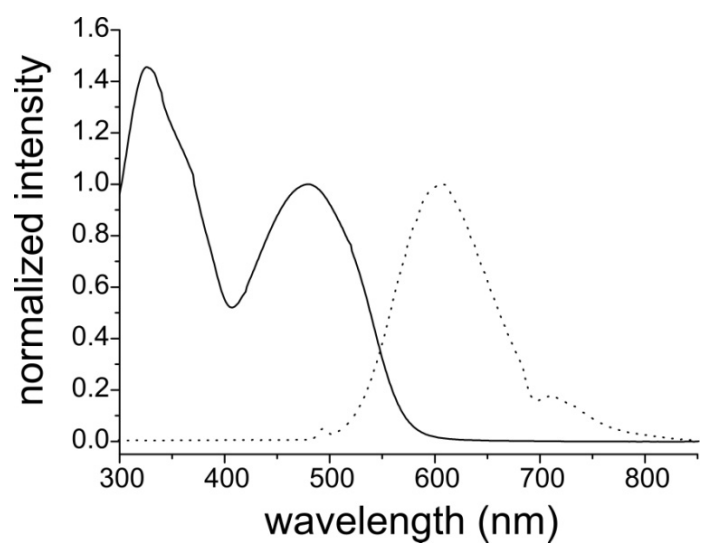

Table 1. Optical $\left(\mathrm{CHCl}_{3}\right)$ and electrochemical $\left(\mathrm{CH}_{3} \mathrm{CN}\right)$ properties of DEHT-V-BTD and poly(DEHT-V-BTD).

\begin{tabular}{|c|c|c|c|c|c|c|}
\hline Species & $\begin{array}{c}E_{\text {gap }}^{\text {opt a }} \\
{[\mathrm{eV}]} \\
\end{array}$ & $\begin{array}{c}\mathbf{E}_{\text {onset }}^{\text {ox b }} \\
{[\mathrm{V}]} \\
\end{array}$ & $\begin{array}{c}\text { HOMO }^{\mathrm{c}} \\
{[\mathrm{eV}]}\end{array}$ & $\begin{array}{c}\mathbf{E}_{\text {onset }} \text { red b } \\
{[\mathrm{V}]}\end{array}$ & $\begin{array}{c}\text { LUMO }^{\mathrm{c}} \\
{[\mathrm{eV}]}\end{array}$ & $\begin{array}{c}E_{\text {gap }}^{\text {eld }} \\
{[\mathrm{eV}]}\end{array}$ \\
\hline DEHT-V-BTD & 2.2 & 0.33 & -5.5 & - & - & - \\
\hline Poly(DEHT-V-BTD) & 1.8 & 0.15 & -5.3 & -1.60 & -3.6 & 1.7 \\
\hline
\end{tabular}

Notes: ${ }^{\text {a }}$ Optical bandgap (calculated on the low energetic edge of the absorption spectrum); ${ }^{\mathrm{b}} \mathrm{Vs}_{\mathrm{S}} \mathrm{Fc} / \mathrm{Fc}^{+}$; ${ }^{\mathrm{c}}$ HOMO and LUMO energies calculated as reported in the Experimental Section; ${ }^{\mathrm{d}}$ Difference between HOMO and LUMO energies.

\subsection{Electrochemical Behavior}

The electrodeposition process represents an efficient technique to prepare conducting polymers since it enables charge and morphology control. Figure 4 compares the three different electrochemical techniques that we have applied to growth poly(DEHT-V-BTD) at the FTO surface, namely cyclic voltammetry (CV, Figure 4a), the potentiostatic (PS, Figure 4b), and the pulsed potentiostatic (PPS) method (Figure 4c,d). The electrochemical characterization in monomer free solution of the resulting layers is described in Figure 5. The electropolymerization reaction was firstly investigated by the potentiodynamic $\mathrm{CV}$ technique (Figure 6a). During the first scan the onset of the oxidation process is around $0.33 \mathrm{~V}$ and the peak lies at $0.47 \mathrm{~V}$. In the cathodic branch one peak appears at around $-0.24 \mathrm{~V}$, due to the reduction of the fresh formed layer. Since the oligomer oxidation takes place at lower potential than the monomer polymerization, the onset of the second cycle anodic process (around $0.06 \mathrm{~V}$ ) is lower than in the first scan. Further cycling shows an increase of current due to the oligomers p-doping redox process, pointing out the cycle by cycle growth of the film at the electrode surface. 
Figure 4. Polymerization of DEHT-V-BTD by different electrochemical techniques: (a) three cyclic voltammetry (CV) cycles; (b) potentiostatic (PS) at $0.39 \mathrm{~V}$ for $100 \mathrm{~s}$ : current/time (black) and charge/time (red) profiles; (c) first six steps of the pulsed-potentiostatic (PPS): current/time (black) and potential/time (red) profiles; and (d) PPS: current/time (black) and charge/time (red) profiles.
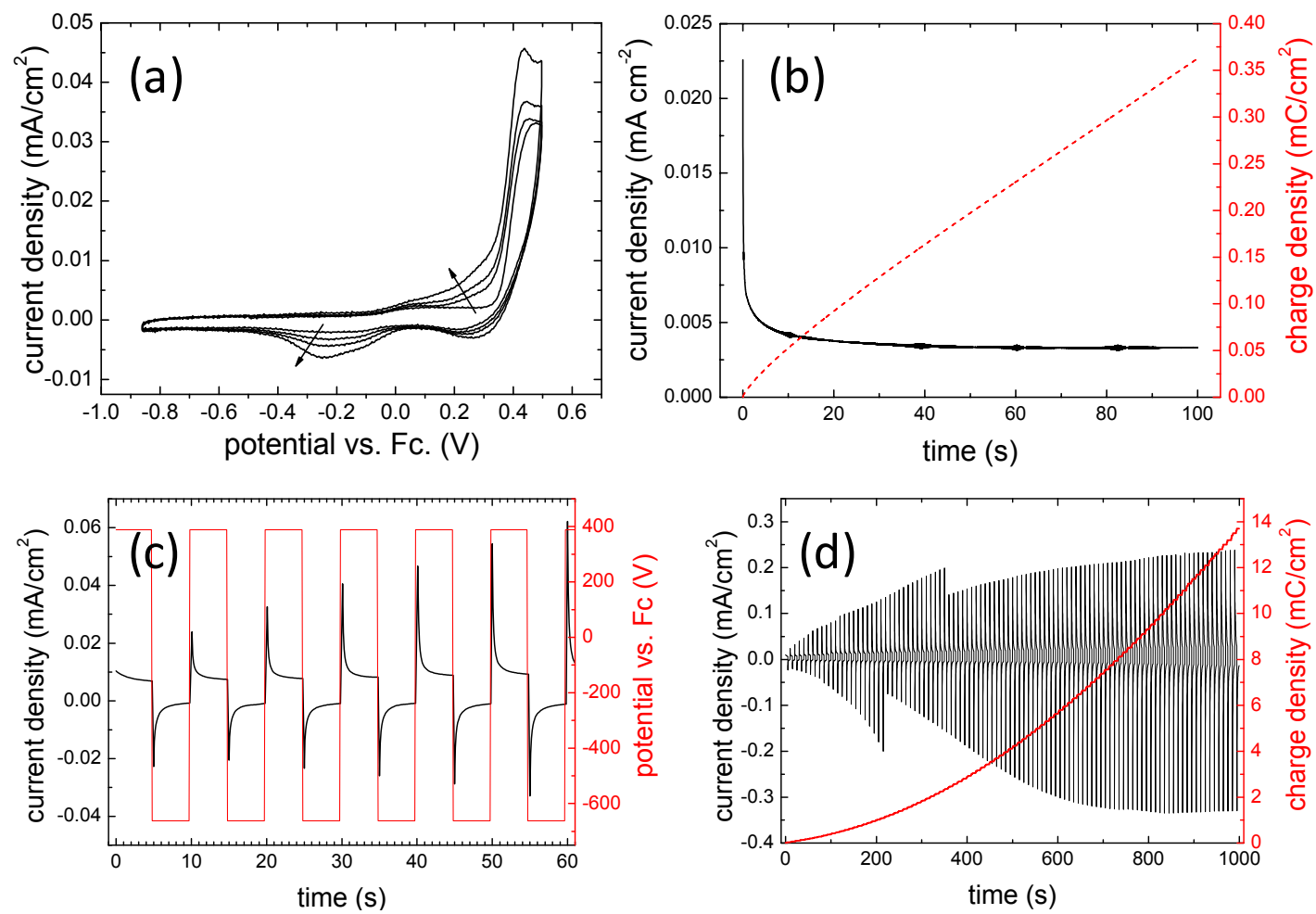

Figure 5. CVs $(20 \mathrm{mV} / \mathrm{s})$ of different poly(DEHT-V-BTD) in monomer free solution: sample obtained by: (a) CV; (b) PS; (c) and (d) PPS.

Figure 6. Spectroelectrochemistry in different potential regions of a poly(DEHT-V-BTD) film prepared by PPS: (a) oxidation of the neutral polymer; (b) reduction of the oxidized polymer; (c) reduction of the neutral polymer; and (d) oxidation of the reduced polymer. OCP refers to the open circuit potential spectrum.


The electrochemical characterization of the resulting film in monomer-free electrolyte (Figure 5a) shows an oxidation process which becomes stable after the first cycle with an onset at $-0.07 \mathrm{~V}$. Upon inverting the potential wave, the current drops immediately to negative values, as expected for semiconducting polymeric materials. Forcing the potential to more negative values a second current wave can be observed at $-1.80 \mathrm{~V}$, attributed to oligomers reduction (n-doping) which appears as a partially reversible process when the potential is stepped back in the anodic direction. However, the film appears to be very thin (see also the current density value) and spread in a non-homogeneous manner onto the FTO substrate; the optical quality was not considered good enough for further electro-optical characterizations.

In order to improve the polymerization process and the layer homogeneity as well as to increase the amount of active material, both potentiostatic (PS) and the pulsed potentiostatic techniques (PPS) were carried out for the polymerization process. In the PS method a single potentiostatic pulse $(0.39 \mathrm{~V} v s$. Fc) was applied for $100 \mathrm{~s}$. The corresponding current output is shown in Figure $4 \mathrm{~b}$ together with the nominal charge density as calculated according to the Faraday law. The applied potential was chosen close to the peak position observed in the monomer CV (Figure 4a). The corresponding initial current density $\left(>20 \mu \mathrm{A} / \mathrm{cm}^{2}\right)$ is in agreement with the current observed by $\mathrm{CV}$. However, the current drops to very low values $\left(<5 \mu \mathrm{A} / \mathrm{cm}^{2}\right)$ after few seconds and the corresponding charge growth results very slow. The obtained layers show electrochemical properties similar to the CV samples (Figure 5b) 
though thinner (see the corresponding current densities in Figure 5), and present an even worse homogeneous character than the polymer produced by $\mathrm{CV}$. We have previously observed that vinylene-spaced monomers are more difficult to electropolymerize compared to conventional D-A-D systems [10]. Moreover, the polymerization of $\mathrm{D}-\mathrm{A}-\mathrm{D}$ monomers is intrinsically more arduous compared to simpler heteroaromatic rings, since the radical cation formed during the first oxidative step can delocalize the unpaired electron on the central acceptor group, causing an additional kinetic barrier for the successive chemical coupling step at the terminal sites. This effect increases as the electron-withdrawing power of the central acceptor unit is stronger. Indeed, the pyridine-EDOT donor-acceptor polymers were electropolymerized using the PS technique [10]. To overcome such problems and produce homogeneous and thicker layers, we have therefore decided to apply the pulsed potentiostatic method (PPS). A detailed discussion of the application of pulsed techniques to the growth of conducting polymers has been reported [17].

In PPS mode (see Figure 4c for the first six steps) the potential was first set to a value higher than the oxidation potential onset of the monomer $(0.39 \mathrm{~V}$, close to the current maximum in $\mathrm{CV})$ for a specific period of time $(5 \mathrm{~s})$ and then switched to a lower value, where no monomer oxidation can take place $(-0.66 \mathrm{~V}, 5 \mathrm{~s})$. The lower potential value was chosen in order to change to oxidation state of the polymer and to promote morphological rearrangements in the polymeric backbone, which may be beneficial for the successive steps of polymer formation [17]. The process was iterated 100 times in order to grow a sufficient amount of polymer. Figure $4 d$ shows the current output together with the nominal polymerization charge density as calculated from the Faraday law, considering that the polymer growths only during the higher voltage step, whereas the lower voltage step does not affect the polymerization charge. The calculated deposition charge is $13.7 \mathrm{mC} / \mathrm{cm}^{2}$ (100 iterations), which is much larger than that which would be achievable by a normal PS deposition. Figures $5 \mathrm{c}$ shows the electrochemical behavior in the full potential range. The current density is higher than the layers produced by CV or PS as expected from the larger deposition charge. The CV profiles stabilize after five cycles and clearly show the $\mathrm{p}$ - and n-doping processes which take place above $0.15 \mathrm{~V}$ (current peak lying at $0.36 \mathrm{~V}$ ) and below $-1.6 \mathrm{~V}$ (current peak at $-1.84 \mathrm{~V}$ ), respectively. During the first cycles, the $\mathrm{p}$ de-doping process (the polymeric backbone switches from the positive to the neutral state) shows a weak charge trapping effect at around $-0.30 \mathrm{~V}$. This peak disappears after five cycles, suggesting that a morphological rearrangement occurs in the layer. If the potential is scanned in the p- and n-doping regions separately (see Figure 5d), the CV profiles are stable since the first cycle and the charge trapping effect does not disappear. The reduction of the neutral poly(DEHT-V-BTD) towards the negative charged state and its re-oxidation is characterized by redox sharp peaks separated by $\sim 100 \mathrm{mV}$, showing the localized character of the generated charged species and the efficient reversibility of the process. The charge related to the low potential process $(<-1.5 \mathrm{~V})$ is about 1.5 times higher than that of the high potential process $(>-0.5 \mathrm{~V})$. Albeit the value maybe is strongly affected by the measurement cutoffs, this finding may be due to the fact that the cathodic process requires one electron per each monomeric unit while the p-doping process involves more units. The hysteresis of the anodic process is usually related to the strong conformational change taking place when the neutral chain is oxidized, while the reduction has more molecular character being the inserted charge localized on the acceptor groups. The HOMO and LUMO values obtained from CV were calculated to be -5.3 and $-3.6 \mathrm{eV}$, respectively, with a band gap of $1.7 \mathrm{eV}$. Table 1 summarizes the 
optical and electrochemical findings in terms of energy levels for both the monomer and the corresponding polymer obtained by PPS.

\subsection{In Situ UV-Vis Spectroscopy}

In order to better elucidate the nature of the doping processes of the polymers and get deeper details on the involved charge carriers, we have performed in situ UV-Vis spectroscopy (spectroelectrochemistry). When the neutral backbone organic chain is oxidized, polaronic species are firstly formed. In oligothiophene based systems, one polaronic transition is localized in the range 750-1000 nm, depending on the extensions of chain lengths [18]. As the oxidative process proceeds, bipolaronic states are obtained. The aforementioned transition decreases in intensity while a new absorption band appears at lower energies $(>1500 \mathrm{~nm})$. The spectroelectrochemical characterization was performed on the PPS obtained layer at various bias potentials (Figure 6) after several stabilization CV cycles in monomer free solution. The end point of the last $\mathrm{CV}$ cycle was chosen so to drive the polymer in the neutral state. In the corresponding spectrum (Open Circuit Potential, OCP, curve in Figure 6a) two main absorption processes were recorded in the Vis region at 515 and at a value below $340 \mathrm{~nm}$. Both transitions are also present in the spectrum of the monomer. The high energy band may be attributed to the to a simple $\pi-\pi^{*}$ transition which is polarized along the chain axis of the molecule. As expected, the CT band is bathochromically shifted on going from the monomer to the oligomeric layer because of increased $\pi$-conjugation path and decreased HOMO-LUMO gap. However, the small shift of the long-wavelength absorption band from the monomer to the "polymer" suggests that oligomers with a low number of units have been generated during electropolymerization.

The optical bandgap, estimated from the low energetic edge of main absorption band (515 $\mathrm{nm})$ in the OCP spectrum, is in agreement with the electrochemical value. When oxidative potentials are applied, a new band appears at $740 \mathrm{~nm}$, ascribed to the formation of polaronic states inside the bandgap, and the CT transition at $515 \mathrm{~nm}$ decreases in intensity (Figure 6a). Curiously, further oxidation processes increase the polaronic rather than the bipolaronic bands. This behavior, not present in the case of conventional D-A-D polymers, has been previously observed though never highlighted $[5,10,12,19,20]$. Thus the presence of vinylene spacers either apparently inhibits the formation of bipolaronics bands or shifts them at lower energy. Anyhow, the absorption due to polaronic species does not decrease with the increase of the doping level. The optical change upon oxidation is reversible; in fact, upon reducing the oxidized layer to its neutral state the optical spectrum accordingly changes (Figure 6b). The reduction of the neutral layer at potential close to the $\mathrm{CV}$ cathodic current onset $(-1.61 \mathrm{~V})$ results in a lower intensity of the CT band located at $515 \mathrm{~nm}$ and in the formation of a new weak transitions at lower energies (690 nm, see Figure 6c), which is attributed to the formation of negatively charged polarons. As in the case of the p-doping process, the further reduction of the system towards the $\mathrm{CV}$ cathodic peak $(-1.8 \mathrm{~V})$ is followed by a significant increase of the intensity of this band, with no evidence of the formation of negative bipolaronic species. The reversibility of the process is also confirmed by the spectra evolution during layer re-oxidation (Figure 6d). The very faint color change observed (poor electrochromic properties, i.e., very low optical contrast) is due to two main reasons: the low layer thickness (the maximum relative change in the absorption values is just 0.03 in Figure 6$)$ and the range $(\lambda>750 \mathrm{~nm})$ of the largest optical changes. 


\section{Conclusions}

A new monomer, containing an unprecedented combination of strong donor (DEHT), acceptor (BTD), and vinylene spacer, has been prepared to get insights into vinylene-linked donor-acceptor-donor low bandgap semiconducting polymers. The monomer showed an optical energy gap of $2.2 \mathrm{eV}$ and an onset for oxidation at $0.33 \mathrm{~V} v s$. Fc. The corresponding polymers were obtained by electrochemical polymerization using cyclic voltammetry either under potentiostatic conditions or by the pulse potentiostatic technique. The latter proved to be an efficient method to produce layers with sufficient amounts of material and reversible electrochemical and spectroelectrochemical characteristics. The polymer shows HOMO, LUMO, and bandgap energies of $-5.3,-3.6$, and $1.7 \mathrm{eV}$, respectively. These values are promising for an efficient use as a p-conducting layer in BHJ photovoltaic devices in combination with PCBM. In comparison with vinylene-linked donor-acceptor polymers carrying the pyridine ring as the $\pi$-electron poor moiety, the new polymer exhibits a significantly lower LUMO level, thanks to the presence of the stronger acceptor BTD group compared the pyridine ring (see Table 2). The lower LUMO energy yields a more proper offset of $0.7 \mathrm{eV}$ respect to the LUMO of PCBM (measured under same conditions), which thus results in a potential lower energy loss between the absorbed photon and the device voltage. In addition, the lower LUMO energy allows for a smaller bandgap, which might yield a more efficient light harvesting and, accordingly, a higher device current. Efforts are under way to synthesize via the chemical route and investigate the corresponding polymer in a BHJ photovoltaic cell in order to assess the promisingly good current and voltage parameters.

Table 2. Highest Occupied Molecular Orbital (HOMO), Lowest Unoccupied Molecular Orbital (LUMO), and bandgap energies of poly(DEHT-V-BTD), in comparison with other D-A-D vinylene-linked polymers and PCBM.

\begin{tabular}{cccc}
\hline Species & HOMO [eV] & LUMO [eV] & Bandgap [eV] \\
\hline PCBM & \multicolumn{4}{c}{-4.3} \\
poly(DEHT-V-BTD) & -5.3 & -3.6 & 1.7 \\
poly(2,5-pyridine-V-PEDOT) ${ }^{\text {a }}$ & -5.2 & -3.2 & 2.0 \\
poly(2,5-pyridine-V-pyrrole) ${ }^{\mathrm{b}}$ & -5.4 & -3.2 & 2.2 \\
\hline \multicolumn{4}{c}{ Notes: $^{\mathrm{a}}$ From [10]; ${ }^{\mathrm{b}}$ From [9]. }
\end{tabular}

Spectroelectrochemical studies have established that charge polaronic species are formed at a low doping level and are stable in the full potential range during the layer oxidation and reduction steps, a behavior which seems closely related to the specific chemical nature of the monomeric building block.

\section{Acknowledgments}

Authors are grateful to Fondazione Cariplo for the financial funding (Grant 2007-5085).

\section{Conflict of Interest}

The authors declare no conflict of interest. 


\section{References}

1. Po, R.; Maggini, M.; Camaioni, N. Polymer solar cells: Recent approaches and achievements. J. Phys. Chem. C 2010, 114, 695-706.

2. Havinga, E.E.; ten Hoeve, W.; Wynberg, H. A new class of small band gap organic polymer conductors. Polym. Bull. 1992, 29, 119-126.

3. Brabec, C.J.; Sariciftci, N.S.; Hummelen, J.C. Plastic solar cells. Adv. Funct. Mater. 2001, 11, 15-26.

4. Yamamoto, T.; Zhou, Z.-H.; Kanbara, T.; Shimura, M.; Kizu, K.; Maruyama, T.; Nakamura, Y.; Fukuda, T.; Lee, B.-L.; Ooba, N.; et al. $\pi$-Conjugated donor-acceptor copolymers constituted of $\pi$-excessive and $\pi$-deficient arylene units. Optical and electrochemical properties in relation to CT structure of the polymer. J. Am. Chem. Soc. 1996, 118, 10389-10399.

5. Thompson, B.C.; Kim, Y.-G.; McCarley, T.D.; Reynolds, J.R. Soluble narrow band gap and blue propylenedioxythiophene-cyanovinylene polymers as multifunctional materials for photovoltaic and electrochromic applications. J. Am. Chem. Soc. 2006, 128, 12714-12725.

6. Zotti, G.; Zecchin, S.; Schiavon, G.; Berlin, A.; Penso, M. Ionochromic and potentiometric properties of the novel polyconjugated polymer from anodic coupling of 5,5'-bis(3,4(ethylenedioxy)thien-2-yl)-2,2'-bipyridine. Chem. Mater. 1999, 11, 3342-3351.

7. Groenendaal, L.; Zotti, G.; Aubert, P.-H.; Waybright, S.M.; Reynolds, J.R. Electrochemistry of poly(3,4-alkylenedioxythiophene) derivatives. Adv. Mater. 2003, 15, 855-879.

8. Beaujuge, P.M.; Ellinger, S.; Reynolds, J.R. The donor-acceptor approach allows a black-to-transmissive switching polymeric electrochrome. Nat. Mater. 2008, 7, 795-799.

9. Abbotto, A.; Herrera Calderon, E.; Manfredi, N.; Mari, C.M.; Marinzi, C.; Ruffo, R. Vinylene-linked pyridine-pyrrole donor-acceptor conjugated polymers. Synth. Met. 2011, 161, 763-769.

10. Abbotto, A.; Calderon, E.H.; Dangate, M.S.; de Angelis, F.; Manfredi, N.; Mari, C.M.; Marinzi, C.; Mosconi, E.; Muccini, M.; Ruffo, R.; et al. Pyridine-edot heteroarylene-vinylene donor-acceptor polymers. Macromolecules 2010, 43, 9698-9713.

11. Roncali, J. Electrogenerated functional conjugated polymers as advanced electrode materials. J. Mater. Chem. 1999, 9, 1875-1893.

12. Mei, J.; Heston, N.C.; Vasilyeva, S.V.; Reynolds, J.R. A Facile Approach to defect-free vinylene-linked benzothiadiazole-thiophene low-bandgap conjugated polymers for organic electronics. Macromolecules 2009, 42, 1482-1487.

13. Beaujuge, P.M.; Subbiah, J.; Choudhury, K.R.; Ellinger, S.; McCarley, T.D.; So, F.; Reynolds, J.R. Green Dioxythiophene-benzothiadiazole donor-acceptor copolymers for photovoltaic device applications. Chem. Mater. 2010, 22, 2093-2106.

14. King, G.; Higgins, S.J. Synthesis and characterisation of novel substituted benzo[c]thiophenes and polybenzo[c]thiophenes: Tuning the potentials for $\mathrm{n}$ - and p-doping in transparent conducting polymers. J. Mater. Chem. 1995, 5, 447-455.

15. Bard, A.J.; Faulkner, R.L. Electrochemical Methods, 2nd ed.; John Wiley and Sons: New York, NY, USA, 2001; p. 54.

16. Sawyer, D.T.; Sobkowiak, A.; Roberts, J.L., Jr. Electrochemistry for Chemists, 3rd ed.; John Wiley and Sons: New York, NY, USA, 1995; p. 203. 
17. Schuhmann, W.; Kranz, C.; Wohlschlaeger, H.; Strohmeier, J. Pulse technique for the electrochemical deposition of polymer films on electrode surfaces. Biosens. Bioelectron. 1997, 12, 1157-1167.

18. Van Haare, J.A.E.H.; Havinga, E.E.; van Dongen, J.L.J.; Janssen, R.A.J.; Cornil, J.; Brèdas, J.-L. Redox states of long oligothiophenes: Two polarons on a single chain. Chem. Eur. J. 1998, 4, $1509-1522$.

19. Galand, E.M.; Kim, Y.-G.; Mwaura, J.K.; Jones, A.G.; McCarley, T.D.; Shrotriya, V.; Yang, Y.; Reynolds, J.R. Optimization of narrow band-gap propylenedioxythiophene:cyanovinylene copolymers for optoelectronic applications. Macromolecules 2006, 39, 9132-9142.

20. Beaujuge, P.M.; Vasilyeva, S.V.; Ellinger, S.; McCarley, T.D.; Reynolds, J.R. Unsaturated linkages in dioxythiophene-benzothiadiazole donor-acceptor electrochromic polymers: The key role of conformational freedom. Macromolecules 2009, 42, 3694-3706.

(C) 2013 by the authors; licensee MDPI, Basel, Switzerland. This article is an open access article distributed under the terms and conditions of the Creative Commons Attribution license (http://creativecommons.org/licenses/by/3.0/). 